\title{
Características populacionais de Microphrys bicornutus (Brachyura, Mithracidae) no fital Halimeda opuntia (Chlorophyta, Halimedaceae), em área recifal submetida à visitação humana, em João Pessoa, Paraíba
}

\author{
Jefferson B. Batista ${ }^{1}$, Rosa M. V. Leonel ${ }^{2} \&$ Marcos A. J. Costa $^{1}$ \\ 1. Programa de Pós-Graduação em Ciências Biológicas (Zoologia), Departamento de Sistemática e Ecologia, Centro de Ciências Exatas \\ e da Natureza, Universidade Federal da Paraíba, 58059-900 João Pessoa, PB, Brasil. (biojef13@yahoo.com.br). \\ 2. Departamento de Sistemática e Ecologia, Universidade Federal da Paraíba, 58059-900 João Pessoa, PB, Brasil. (rleonel@oi.com.br).

\begin{abstract}
Population characteristics of Microphrys bicornutus (Brachyura, Mithracidae) on the phytal Halimeda opuntia (Chlorophyta, Halimedaceae), on reef area submitted to human visitation, in João Pessoa, Paraíba, Brazil. The aim of this study was to characterize and compare the population structure and dynamic of Microphrys bicornutus Latreille, 1825 on the phytal Halimeda opuntia (Halimedaceae) collected on the reef areas of Picãozinho (visited by tourists) and São Gonçalo (control area), in João Pessoa coast (Northeastern of Brazil), under the influence of environmental conditions and visitant numbers. In both study areas the populations analyzed were composed similarly of mature and immature males and females, with predominance of males and immature animals, and similar size frequency and reproductive period. Sex-ratio, size frequency distribution, and size of the biggest specimens differed from those estimated in others latitudes and habitats for this species. Sex ratio estimation has made evident that independently of maturation stage, males tended to predominate significantly and the proportion of females to decrease with sexual maturation. Without exhibiting influence of the alga biomass, and of the salinity and temperature of the water, significant population variations were associated to the increase of juvenile during rainy periods. The low population density and the largest disproportion of the relationship male: female in Picãozinho subarea with larger flow of people, are suggestive that the trampling of the alga might have induced this population variation.
\end{abstract}

KEYWORDS. Population composition, population density, sex ratio, algae trampling, seasonal effect.

RESUMO. Este trabalho teve como objetivos caracterizar e comparar a estrutura e a dinâmica populacional de Microphrys bicornutus Latreille, 1825 no fital Halimeda opuntia (Halimedaceae) coletado nas formações recifais de Picãozinho (submetida à visitação turística) e São Gonçalo (área controle), na costa de João Pessoa (Nordeste do Brasil), sob influência de fatores ambientais e do número de visitantes. Nas duas áreas de estudo as populações analisadas estiveram compostas por fêmeas e machos maduros e imaturos com significativa predominância de machos e de animais imaturos, frequêencia de tamanho e períodos reprodutivos similares. O tamanho máximo dos exemplares, a frequiência de distribuição de tamanho e a razão sexual diferiram dos resultados obtidos para a espécie em outras latitudes e habitats. Dados de razão sexual evidenciam que independentemente do estágio de maturação, os machos apresentam predominância significativa (RS>1,0), e que a proporção de fêmeas diminui com o amadurecimento sexual. Sem sofrer influência da biomassa da alga, e da salinidade e temperatura da água, variações populacionais significativas foram associadas ao aumento de juvenis durante períodos chuvosos. A baixa densidade populacional e a maior desproporção da relação macho: fêmea em subárea de Picãozinho com maior fluxo de pessoas sugerem que estas variações podem ter sido induzidas pelo pisoteio das algas.

PALAVRAS-CHAVE. Composição populacional, densidade populacional, razão sexual, pisoteio de algas, efeito estacional.

Constituído por macroalgas e gramas marinhas, o fital representa um habitat favorável à reprodução, desenvolvimento e crescimento de diversos invertebrados, sendo uma fonte potencial de alimento tanto para espécies intrínsecas quanto extrínsecas a este ecossistema (MASUnARI \& Forneris, 1981; MASUNARI, 1987), fazendo parte da dieta de peixes de importância econômica e de áreas recifais (PALMA \& OJEDA, 2002; NAKAMURA et al., 2003).

A composição e a densidade populacional da epifauna dos fitais podem ser afetadas por diversos fatores, tais como as características da planta-substrato (e.g. arquitetura, consistência, biomassa e composição química), o comportamento dos animais (e.g. migração, predação e preferência alimentar), variações abióticas (e.g. temperatura, hidrodinamismo, profundidade e teor de sedimento) e a ação antrópica direta (pisoteio) ou indireta (poluição da água) (MuKaI, 1971; Moore, 1973; Montouchet, 1979; Masunari, 1982, 1983, 1987; Edgar, 1983; Coen, 1988; Dubiaski-Silva \& Masunari, 1995; JeRNAKOFF \& NiELSEN, 1998; BROWN \& TAYLOR, 1999;
ECKrich \& Holmquist, 2000; Parker et al., 2001; VAN Alstyne et al., 2001; Chemello \& Milazzo, 2002; NAKAMURA et al., 2003).

Estudos sobre os Brachyura que compõem a epifauna de fitais do litoral brasileiro são ainda escassos, estando principalmente relacionados com a análise da influência do sedimento, do hidrodinamismo e da morfologia do talo sobre a composição e a densidade das espécies (GouvêA \& Leite, 1980; Masunari, 1982; BARBosa \& LEONEL, 2003), havendo uma lacuna no conhecimento da estrutura populacional desses animais neste habitat.

Participando da epifauna de Halimeda opuntia (Halimedaceae) presente nas formações recifais de Picãozinho e São Gonçalo (Paraíba), o caranguejo decorador Microphrys bicornutus Latreille, 1825 representa o Brachyura mais conspícuo e abundante deste fital. Desses dois recifes, Picãozinho se notabiliza por receber contínua visitação turística e São Gonçalo por se manter como uma área praticamente isenta da presença humana. 
Apresentando ampla distribuição geográfica, $M$. bicornutus ocorre no Atlântico Ocidental desde a Carolina do Norte até o sul da Florida (EUA), nas Bermudas, Golfo do México, Antilhas, América Central, Venezuela e, no Brasil, do Maranhão ao Rio Grande do Sul e em Fernando de Noronha (Melo, 1996). No litoral paraibano a presença deste caranguejo tem sido assinalada em recifes de corais e em quase todos habitats de águas rasas (Melo \& Veloso, 2005), incluindo diferentes fitais da região de entre-marés (BARBOSA \& LEONEL, 2003).

Estudos realizados em área de manguezal por LóPEZ Greco et al. (2000), indicam que além da latitude, a temperatura, a salinidade, o espaço disponível e a quantidade e qualidade de alimento disponível podem influir sobre o crescimento e a reprodução de $M$. bicornutus em populações muito próximas. Sendo uma espécie onívora de hábito generalista, $M$. bicornutus apresenta dieta alimentar composta por macroalgas e invertebrados, forrageia conforme a disponibilidade dos recursos alimentares e as marés (KILAR \& Lou, 1984), e tem seu crescimento e reprodução sustentados pela ingestão de poliquetos, esponjas e algas oportunistas (KILAR \& Lou, 1986).

Representando uma primeira contribuição para o conhecimento das características populacionais de $M$. bicornutus no litoral brasileiro, o presente trabalho teve como objetivos, caracterizar e comparar a estrutura e a dinâmica populacional desta espécie no fital $H$. opuntia oriundo dos recifes de Picãozinho e São Gonçalo, sob influência da biomassa da alga, de fatores abióticos e da presença de turistas.

\section{MATERIAL E MÉTODOS}

Localizadas próximo à orla litorânea da cidade de João Pessoa, Paraíba, Nordeste do Brasil, as formações

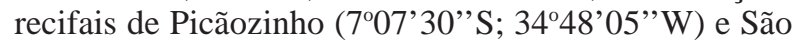
Gonçalo (706'42”'S; 3448'48”'W) (Fig. 1), se encontram separadas por uma distância de $459 \mathrm{~m}$. Durante as marés baixas suas plataformas afloram à superfície, exibindo piscinas naturais contendo diferentes organismos marinhos. Entre as macroalgas presentes, $H$. opuntia constitui uma das espécies mais abundantes e conspícuas nas duas formações. Em Picãozinho, a ancoragem dos barcos ocorre em um canal (canal principal) que, cortando parcialmente a formação no sentido oeste-leste, oferece facilidade de desembarque aos passageiros.

As condições climáticas da região são típicas do litoral nordeste do Brasil, com um período de seca ou estiagem (de setembro a fevereiro) e por um período chuvoso (de março a agosto). Com índice pluviométrico anual maior que $1.250 \mathrm{~mm}$, a região apresenta temperaturas mínima e máxima de $24 \mathrm{e} 36^{\circ} \mathrm{C}$, respectivamente (NIMER, 1977).

Durante o período de setembro de 2002 a agosto de 2004, valores mensais da temperatura e da salinidade da água foram medidos em todos os pontos de coleta das duas áreas com um termômetro de mercúrio e um refratômetro, respectivamente. Valores pluviométricos foram obtidos na estação do INMET $\left(07^{\circ} 05^{\prime} 34^{\prime \prime}\right.$; $34^{\circ} 49^{\prime} 59^{\prime \prime} \mathrm{W}$ ), pelo Laboratório de Meteorologia e Sensoriamento Remoto da Universidade Federal de Campina Grande. Quinzenalmente foi feita a contagem do número de visitantes à medida que ia ocorrendo seu desembarque, observando-se o percurso realizado pelas pessoas em Picãozinho.

Para poder contemplar a dimensão da área, a atividade dos visitantes ao longo da plataforma recifal e uniformizar o esforço de coleta em relação a São Gonçalo (área controle), em Picãozinho foram demarcadas três subáreas: Sul, situada na face voltada para o continente e localizada ao sul do canal principal; Norte, situada na face voltada para o continente e localizada ao norte do canal principal; Leste, na face voltada para o oceano. Devido ao aspecto alongado de São Gonçalo, as coletas foram realizadas ao longo de seu maior eixo.

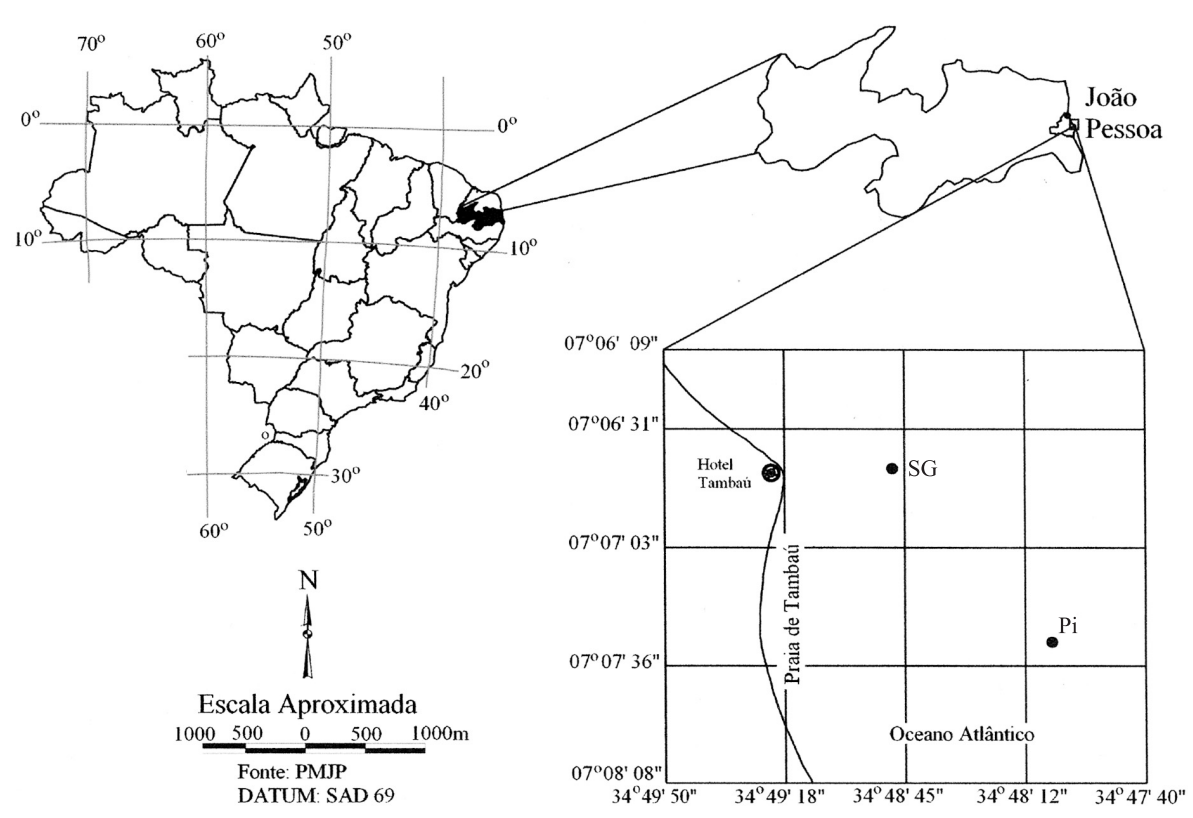

Fig. 1. Mapa do litoral da cidade de João Pessoa, sinalizando a localização das áreas de coleta das formações recifais de Picãozinho (Pi) e São Gonçalo (SG). 
Durante o período citado, três amostras mensais de $H$. opuntia foram coletadas aleatoriamente em bancos submersos nas piscinas naturais, nas três subáreas de Picãozinho e em São Gonçalo. Após serem cobertas por um saco plástico, as algas contidas por um delimitador de $200 \mathrm{~cm}^{2}(13,8 \mathrm{~cm}$ x $14,5 \mathrm{~cm})$ foram totalmente retiradas do substrato com uso de uma espátula.

Em laboratório, as amostras coletadas foram mantidas separadamente em bandejas contendo água do mar ( salinidade $=35$ ) e arejamento contínuo, até posterior triagem dos animais sob lupa manual, com pinça e/ou pipeta Pasteur. Os animais foram fixados em formalina a $4 \%$ em água do mar a 35 e preservados em álcool $70 \%$. A identificação dos espécimes foi baseada em Melo (1996) e em espécimes tombados na Coleção de Invertebrados Paulo Young (CIPY) (UFPB-5472), do Departamento de Sistemática e Ecologia/UFPB. Os espécimes analisados neste trabalho foram depositados na mesma coleção, lotes 5571 a 5579.

Após a triagem dos animais, as amostras de $H$. opuntia foram previamente secadas em papel filtro e levadas à estufa a $80^{\circ} \mathrm{C}$ durante 48 horas até obtenção de peso constante. $\mathrm{O}$ peso seco das amostras foi medido em uma balança de precisão e os valores de biomassa calculados de acordo com a fórmula: Biomassa $=$ peso $\operatorname{seco}(\mathrm{g}) /$ área do quadrado amostral $\left(\mathrm{cm}^{2}\right)$.

A composição populacional de $M$. bicornutus presente em cada ponto de coleta foi analisada de acordo com o sexo e o estágio de maturação sexual. A identificação dos sexos foi baseada em Melo (1996) e os estágios de maturidade das fêmeas seguiu HARTNOLL (1963) e Alunno-Bruscia \& SAINTE-Marie (1998).

A triagem de machos maduros e imaturos foi feita sob microscopia estereoscópica, com base em critérios morfológicos estabelecidos por HARTNOLL (1965) e critérios morfométricos, considerando como: (i) imaturos, os animais que apresentavam duto espermático achatado, translúcido e com comprimento inferior ao da placa abdominal e, (ii) maduros, os animais que apresentavam duto espermático intumescido com coloração branca opaca e comprimento similar ao da placa abdominal.

A ocorrência de machos e de fêmeas em diferentes estágios de maturação sexual foi analisada de acordo com os períodos estacionais da região e a densidade populacional média mensal (número de espécimes/área do quadrado) de cada ponto de coleta, calculada segundo MASUNARI (1982).

Determinado o comprimento da carapaça segundo HaRTNOll (1963), os espécimes foram agrupados em classes de tamanho com amplitude de 1,0 mm. Todas as medidas foram feitas sob microscopia estereoscópica, utilizando um paquímetro com precisão de $0,01 \mathrm{~mm}$.

Após confirmação da normalidade da distribuição dos dados pelos testes de Kolmogorov-Smirnoff e ShapiroWilk, foram realizados testes de estatística paramétrica, utilizando o programa Statistica for Windows, versão 4.0. A homocedasticidade dos dados foi confirmada pelo teste de Levene $(\alpha<0,01)$ (STATSOFT, 1998).

A razão sexual anual de animais imaturos e maduros foi calculada segundo GROTTA \& LUNETTA (1980). Diferenças foram verificadas através do teste do qui-quadrado $(\mathrm{p}<0,05)$.

As populações de $M$. bicornutus coletadas em Picãozinho e São Gonçalo foram analisadas pela ANOVA $(\mathrm{p}<0,05)$ seguida pelo teste de Tukey no tratamento de resultados significativos, verificando a existência de diferenças populacionais quanto ao número mensal de espécimes procedentes de cada ponto de coleta; de espécimes coletados nos períodos de estiagem e de chuva de cada ano de estudo e mensal de machos e de fêmeas em cada estágio de maturação sexual.

Machos e fêmeas em diferentes estágios de maturação, foram analisados pelo teste $t$, comparando o número de espécimes presentes em cada ano de estudo e nos períodos de estiagem e de chuva.

Foram analisadas as correlações entre o número mensal de espécimes das populações de Picãozinho e de São Gonçalo e o número de machos e de fêmeas (imaturos e maduros) presentes nas duas formações com os valores mensais médios de salinidade e temperatura da água; da biomassa da alga e do número de visitantes (Pearson; $\mathrm{p}<0,05)$.

Os valores médios mensais da biomassa, da temperatura e salinidade da água obtidos em cada ponto de coleta foram submetidos à análise de variância.

\section{RESULTADOS}

Do total de 632 espécimes de $M$. bicornutus coletados, 285 eram fêmeas e 347 eram machos (Tab. I).

O comprimento da carapaça dos espécimes variou de 1 a 11 mm em Picãozinho e de 1 a 9 mm em São Gonçalo (Fig. 2). Nas duas áreas de estudo, o maior número de espécimes ficou compreendido na faixa de tamanho de 2,1 a $5 \mathrm{~mm}$.

Em todos os pontos de coleta, as populações de M. bicornutus estiveram compostas por machos imaturos e maduros e por fêmeas imaturas e maduras (ovígeras e não ovígeras) (Figs. 3-6). Considerando a participação percentual destes indivíduos verifica-se que com uma variação de 51 (Picãozinho Norte) a 57\% (Picãozinho Leste), os machos predominaram em todos os pontos de coleta; variando de 68\% (Picãozinho Sul) a 75\% (São Gonçalo) os animais imaturos compuseram a maior parcela populacional; entre as fêmeas maduras, as ovígeras predominaram (6 a 10\%) em relação às não ovígeras (2 a $5 \%)$. O número de espécimes em cada ponto de coleta e o de machos e fêmeas em diferentes estágios de maturação não diferiu significativamente entre os pontos de coleta $(\mathrm{F}=1,26 ; \mathrm{gl}=22 ; \mathrm{p}>0,05)$ e o número de machos imaturos foi significativamente maior que o de machos maduros e o de fêmeas maduras $(F=4,23 ; \mathrm{gl}=22 ; \mathrm{p}<0,05)$. $\mathrm{O}$ número de fêmeas imaturas foi maior que o de fêmeas maduras,

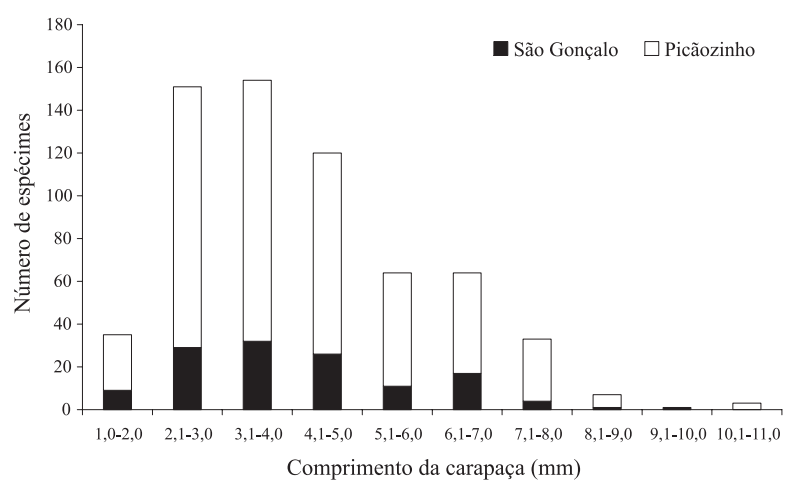

Fig. 2. Número de espécimes de Microphrys bicornutus Latreille, 1825 coletados no fital Halimeda opuntia (Halimedaceae) em Picãozinho e São Gonçalo, Paraíba, de setembro de 2002 a agosto de 2004, de acordo com o comprimento da carapaça. 
Tabela I. Número de fêmeas e de machos imaturos e maduros de Microphrys bicornutus Latreille, 1825, coletados no fital de Halimeda opuntia (Halimedaceae), em Picãozinho e São Gonçalo, Paraíba, de setembro de 2002 a agosto de 2004.

\begin{tabular}{|c|c|c|c|c|c|c|c|}
\hline Ano & Sexo & Estágio de maturação & Picãozinho Leste & Picãozinho Norte & Picãozinho Sul & São Gonçalo & Total \\
\hline \multirow[t]{4}{*}{ Set/02-Ago/03 } & Machos & Maduros (N) & 19 & 10 & 8 & 4 & 41 \\
\hline & & Imaturos $(\mathrm{N})$ & 35 & 33 & 13 & 31 & 112 \\
\hline & Fêmeas & Maduras (N) & 11 & 7 & 3 & 5 & 26 \\
\hline & & Imaturas $(\mathrm{N})$ & 33 & 30 & 12 & 30 & 105 \\
\hline \multirow[t]{4}{*}{ Set/03-Ago/04 } & Machos & Maduros (N) & 9 & 17 & 16 & 10 & 52 \\
\hline & & Imaturos $(\mathrm{N})$ & 56 & 38 & 21 & 27 & 142 \\
\hline & Fêmeas & Maduras (N) & 17 & 14 & 5 & 9 & 45 \\
\hline & & Imaturas $(\mathrm{N})$ & 29 & 39 & 28 & 13 & 109 \\
\hline Total & & & 209 & 188 & 106 & 129 & 632 \\
\hline
\end{tabular}

Tabela II. Porcentagem de fêmeas e machos imaturos e maduros e valores anuais de razão sexual de Microphrys bicornutus Latreille, 1825 em Picãozinho Leste, Norte e Sul e em São Gonçalo, Paraíba, em cada ano de estudo, de setembro de 2002 a agosto de 2004 (F, fêmea; M, macho; PL, Picãozinho Leste; PN, Picãozinho Norte; PS, Picãozinho Sul; RS, razão sexual; SG, São Gonçalo).

\begin{tabular}{|c|c|c|c|c|c|c|c|c|c|c|c|c|}
\hline \multirow[t]{2}{*}{ Estágio/ano } & \multicolumn{2}{|c|}{ PL } & \multicolumn{4}{|c|}{$\mathrm{PN}$} & \multicolumn{2}{|c|}{ PS } & \multicolumn{4}{|c|}{ SG } \\
\hline & $\% \mathrm{M}$ & $\% \mathrm{~F}$ & $\mathrm{RS}$ & $\% \mathrm{M}$ & $\% \mathrm{~F}$ & $\mathrm{RS}$ & $\% \mathrm{M}$ & $\% \mathrm{~F}$ & RS & $\% \mathrm{M}$ & $\% \mathrm{~F}$ & RS \\
\hline \multicolumn{13}{|l|}{ Imaturos } \\
\hline Ano 1 & 51,5 & 48,5 & 1,1 & 52,4 & 47,6 & 1,1 & 52,0 & 48,0 & 1,1 & 50,8 & 49,2 & 1,0 \\
\hline Ano 2 & 65,7 & 34,2 & 1,9 & 49,3 & 50,6 & 0,9 & 52,0 & 48,0 & 1,1 & 67,5 & 32,5 & 2,1 \\
\hline \multicolumn{13}{|l|}{ Maduros } \\
\hline Ano 1 & 63,3 & 36,7 & 1,7 & 58,8 & 41,2 & 1,4 & 72,7 & 27,3 & 2,7 & 44,4 & 55,6 & 0,8 \\
\hline Ano 2 & 34,6 & 65,4 & 0,5 & 54,8 & 45,2 & 1,2 & 76,2 & 23,8 & 3,2 & 52,6 & 47,4 & 1,1 \\
\hline
\end{tabular}

3 Picãozinho Leste

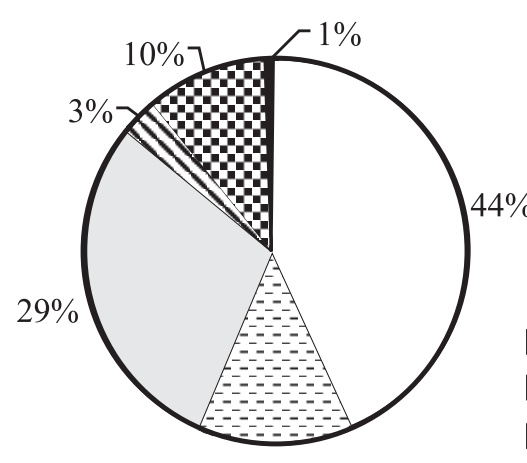

$13 \%$

5

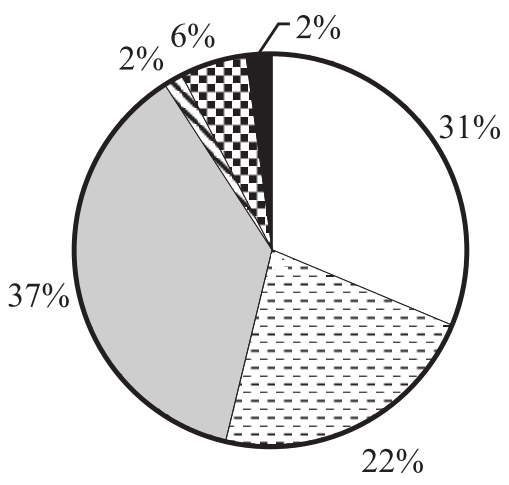

Picãozinho Norte

4

Machos imaturos

GMachos maduros

$\square$ Fêmeas imaturas

\Fêmeas maduras

国Fêmeas ovígeras

Sexo indeterminado

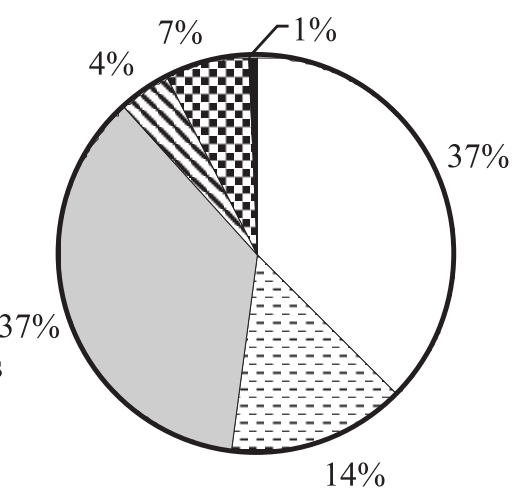

São Gonçalo

6

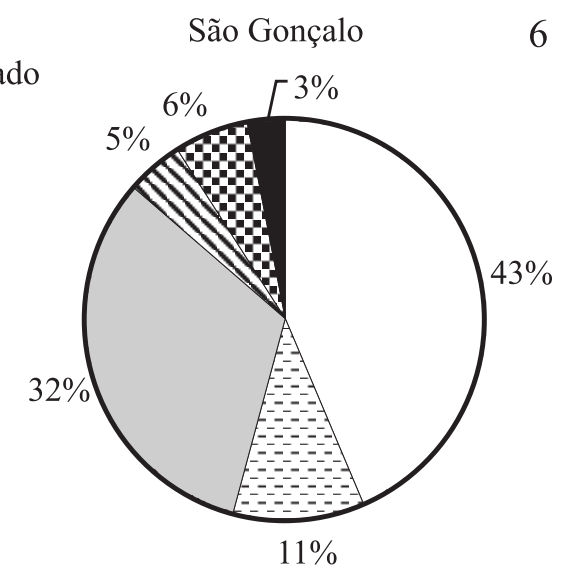

Figs. 3-6. Percentual de machos e de fêmeas de Microphrys bicornutus Latreille, 1825 em diferentes estágios de maturação sexual, nas populações coletadas nas subáreas Leste (3), Norte (4) e Sul (5) de Picãozinho e em São Gonçalo (6), Paraíba, de setembro de 2002 a agosto de 2004. 

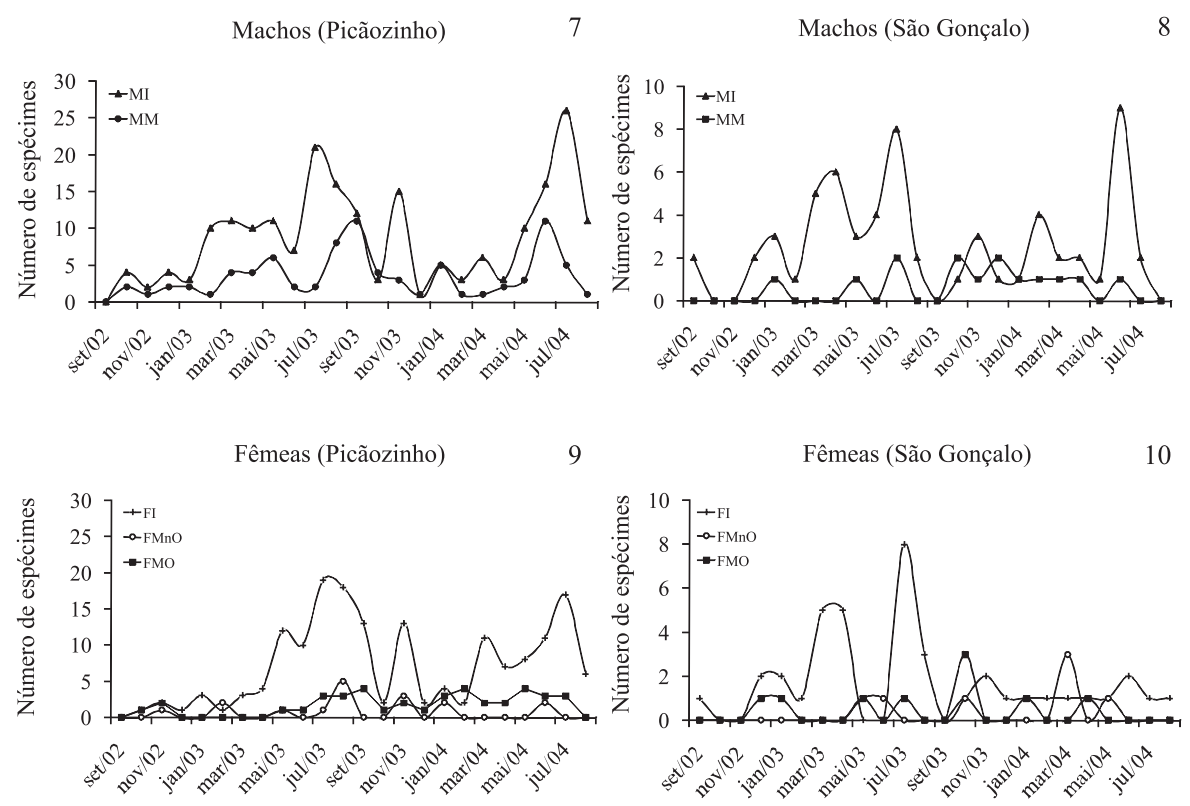

Figs. 7-10. Variação populacional de machos $(7,8)$ e fêmeas $(9,10)$ imaturos e maduros de Microphrys bicornutus Latreille, 1825 em Picãozinho e São Gonçalo, Paraíba, de setembro de 2002 a agosto de 2004 (FI, fêmea imatura; FMnO, fêmea madura não ovígera; FMO, fêmea madura ovígera; MI, macho imaturo; MM, macho maduro).

$2002-2003$
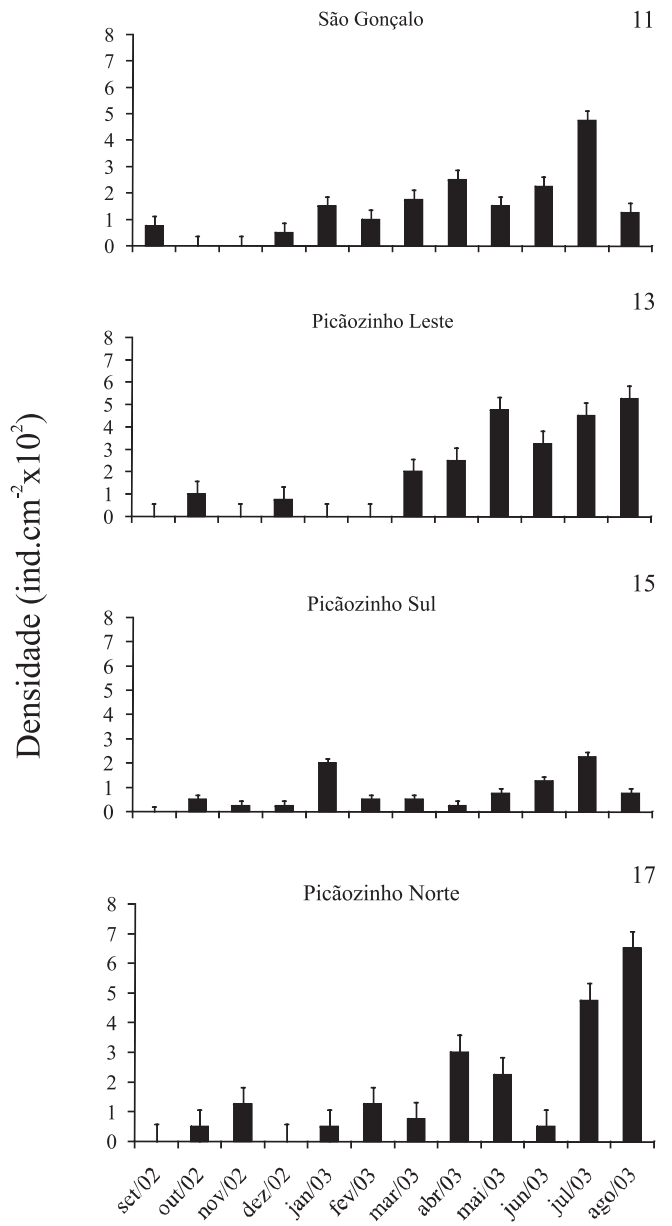

$2003-2004$

São Gonçalo

12
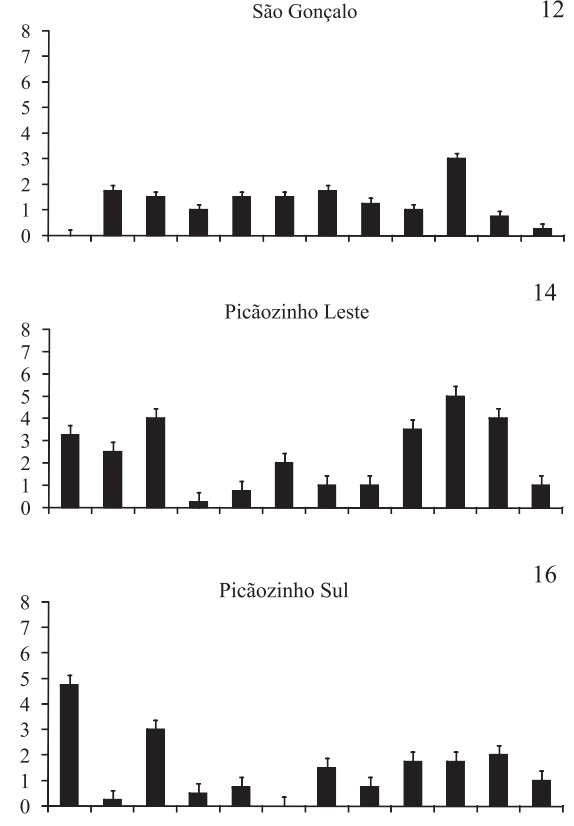

Picãozinho Norte

18

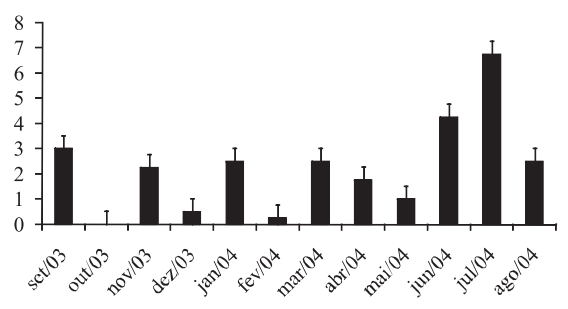

Figs. 11-18. Variação da densidade mensal dos espécimes de Microphrys bicornutus Latreille, 1825 em São Gonçalo $(11,12)$ e nas subáreas Leste $(13,14)$ Sul $(15,16)$ e Norte $(17,18)$ de Picãozinho, Paraíba, em cada ano de estudo, de setembro de 2002 a agosto de 2004. 
tanto em Picãozinho ( $t c=-4,39 ; \mathrm{gl}=46 ; \mathrm{p}<0,05)$, quanto em São Gonçalo ( $t c=-3,32 ; \mathrm{gl}=46 ; \mathrm{p}<0,05)$. Nenhuma diferença significativa foi assinalada entre o número de fêmeas imaturas e maduras e o de machos imaturos e maduros coletados a cada ano $(t c=0,45 ; \mathrm{gl}=94 ; \mathrm{p}<0,05)$.

Embora a variação populacional não tenha se repetido durante os dois anos de estudo (Figs. 7-10), verificou-se que o número de fêmeas e de machos imaturos variou de modo similar em Picãozinho. Fêmeas ovígeras estiveram ausentes nas duas formações em alguns meses do ano, tendendo a serem mais numerosas no período de chuva. Diferenças populacionais significativas foram assinaladas pela ANOVA, evidenciando que o número de espécimes foi maior no período chuvoso do que no período de estiagem ( $\mathrm{F}=6,96$; $\mathrm{gl}=22 ; \mathrm{p}<0,001)$. De acordo com o teste $t$, nos dois anos de estudo o número de fêmeas imaturas $(t c=-3,41 ; \mathrm{gl}=94$; $\mathrm{p}<0,05)$ e de machos imaturos $(t c=-3,11 ; \mathrm{gl}=94 ; \mathrm{p}<0,05)$ foi significativamente maior nos períodos de chuva do que nos períodos de estiagem.

As densidades populacionais de M. bicornutus variaram de zero a 6,75 em Picãozinho e de zero a 4,75 (indivíduos x cm${ }^{-2}$ ) x 100 em São Gonçalo. Em Picãozinho, as maiores densidades populacionais foram assinaladas nos pontos Leste e Norte (Figs. 11-18).

Os dados de razão sexual (Tab. II) denotam significativa superioridade numérica de machos maduros e imaturos em todos os pontos de coleta $\left(\chi^{2}=13,16 ; \mathrm{gl}=22\right.$; $\mathrm{p}<0,05)$ e um aumento da relação machos:fêmeas de acordo com o amadurecimento sexual dos espécimes. A subárea Sul de Picãozinho se notabilizou por apresentar constância anual da relação machos:fêmeas determinada para animais imaturos e por exibir os maiores valores de razão sexual de animais maduros.

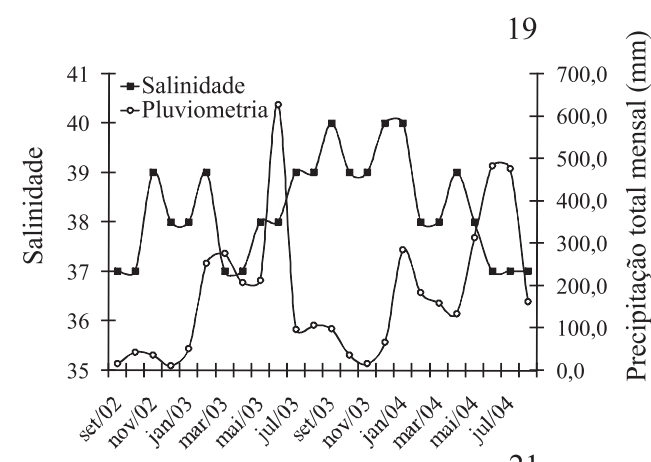

21

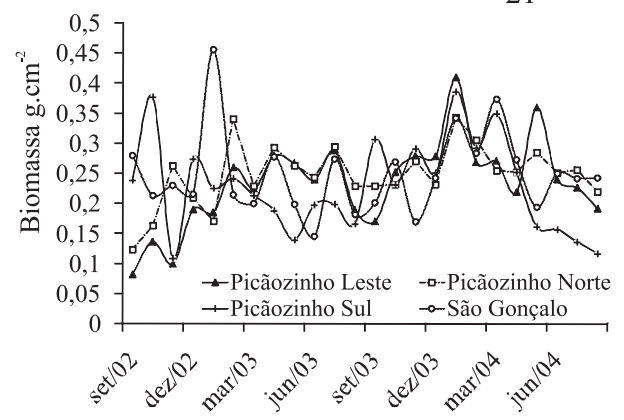

Sem apresentar variação significativa entre os pontos de coleta $(\mathrm{F}=0,45 ; \mathrm{gl}=23 ; \mathrm{P}>0,05)$ (Fig. 19), a salinidade da água oscilou de 37 a 40 em Picãozinho e São Gonçalo, com os valores mais baixos coincidindo com os períodos de chuva (Fig. 20). De modo similar, os valores mensais de temperatura não variaram significativamente, oscilando de 26 a $31^{\circ} \mathrm{C}$ em Picãozinho $(\mathrm{F}=1.999 ; \mathrm{gl}=23 ; \mathrm{p}>0.05)$ e de 26 a $30^{\circ} \mathrm{C}$ em São Gonçalo $(\mathrm{F}=1,54 ; \mathrm{gl}=23 ; \mathrm{p}>0.05)$. Com exceção de dezembro/ 2002 , os valores mais elevados de temperatura da água foram assinalados nos períodos de estiagem. Variando de 15 (setembro/2002) a $626 \mathrm{~mm}$ (junho/2003) (Fig. 19), os dados pluviométricos exibiram variações similares nos dois anos de estudo, estando os períodos de menor e maior pluviosidade associados às estações de estiagem e de chuva, respectivamente.

Embora um padrão de desenvolvimento de $H$. opuntia não tenha sido evidenciado (Fig. 21), observouse uma tendência ao aumento da biomassa nos meses de estiagem. Sem apresentar variação significativa $(\mathrm{F}=0,68$; $\mathrm{gl}=22 ; \mathrm{p}>0,05)$ entre os pontos de coleta, a biomassa da alga variou de 0,16 a $0,36 \mathrm{~g} / \mathrm{cm}^{2}$ em Picãozinho e de 0,15 a $0,46 \mathrm{~g} / \mathrm{cm}^{2}$ em São Gonçalo.

Ao longo do período estudado, o número mensal de visitantes em Picãozinho foi variável (Fig. 22), exibindo maior freqüência de visitantes no período de estiagem. Enquanto nos dois períodos de estiagem analisados, a média mensal de visitantes foi de $228 \pm 90,72$ e de $170 \pm$ 85,03 pessoas, nos períodos de chuva esta média foi de $84 \pm 19,39$ e de $109 \pm 18,06$ pessoas. De acordo com as observações realizadas, o maior fluxo de visitantes ocorreu nas subáreas Sul e Leste de Picãozinho, onde o pisoteio de algas e de animais marinhos é mais acentuado.

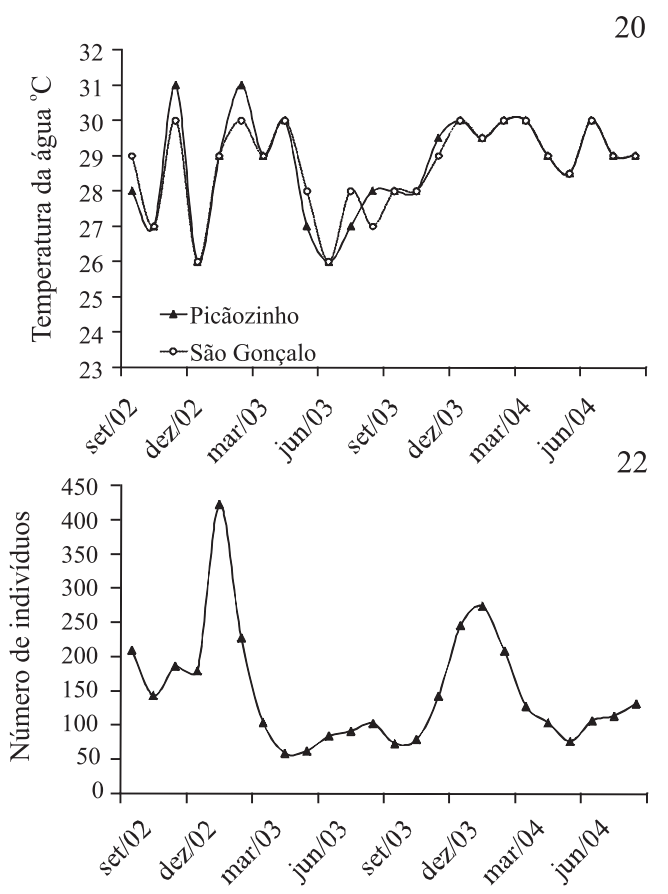

Figs. 19-22. Valores médios mensais da salinidade da água e da precipitação mensal (19), da temperatura da água (20) da biomassa de Halimeda opuntia (Halimedaceae) (21) em Picãozinho e em São Gonçalo, Paraíba, e do número de visitantes em Picãozinho (22). Medidas obtidas de setembro de 2002 a agosto de 2004. 
O número mensal de espécimes de $M$. bicornutus no ponto Leste de Picãozinho apresentou correlação inversa significativa $(\mathrm{r}=-0,90 ; \mathrm{p}<0,05)$ com o número mensal de visitantes. Para as demais variáveis analisadas (biomassa, salinidade e temperatura) nenhuma correlação com o número de espécimes foi estabelecida.

\section{DISCUSSÃO}

Apresentando predominância de machos, a composição bianual das populações estudadas evidenciou que espécimes em todos os estágios de maturação sexual ocorreram em todos os pontos de coleta. Embora a ocorrência de indivíduos em diferentes fases de maturação sexual tenha sido também detectada em população de $M$. bicornutus associada a raízes de Rizophora mangle (López GRECO et al., 2000), diferentemente dos resultados aqui obtidos, naquele habitat houve predominância de fêmeas.

Segundo Hartnoll et al. (1993), a maior produtividade de machos por algumas espécies da família Mithracidae está relacionada com a maior suscetibilidade deste sexo à predação, uma vez que apresentam maior desenvolvimento das quelas e do rostro, camuflagem menos elaborada e maior atividade do que as fêmeas. No entanto, como os resultados aqui obtidos evidenciaram significativa redução do número de machos imaturos e de fêmeas imaturas, admitimos que outros fatores podem ter atuado sobre a diminuição dos dois grupos. Sabendo que peixes micro-carnívoros se alimentam de caranguejos de fitais (NAKAMURA et al., 2003) e que estes organismos ocorrem na área de estudo (RocHA et al., 1998), a predação deve ser considerada como provável fator de interferência nos resultados obtidos. Assim como CARMONA-SuÁrEZ (2007), consideramos que associada ao aumento das temperaturas diárias e à diminuição do teor de oxigênio dissolvido nos bancos de $H$. opuntia durante exposição aérea, a mortalidade dos animais imaturos pode ter sido também induzida pela combinação desses efeitos.

Comparativamente a outras populações, os dados aqui obtidos evidenciaram que dependendo do habitat e da latitude podem ocorrer variações no desenvolvimento e na freqüência de classes de maior tamanho de $M$. bicornutus. Assim, enquanto em $H$. opuntia os maiores exemplares mediram $11 \mathrm{~mm}$ de comprimento, em Rhizophora mangle (Rhizophoraceae) da Venezuela, os maiores espécimes apresentaram um comprimento de carapaça três vezes superior (LóPEz GRECo et al., 2000). Por outro lado, enquanto em $H$. opuntia o tamanho mais freqüente dos espécimes foi de 3 a $5 \mathrm{~mm}$, na grama marinha Thalassia testudinum (Hydrocharitaceae) (CARMONA-SuÁrez, 1992) coletada na Venezuela, esta variação foi de 3 a $9 \mathrm{~mm}$ e em $R$. mangle de 11 a $19 \mathrm{~mm}$ (LóPEZ GRECO et al., 2000). Embora diferenças de tamanho entre populações de caranguejos-aranha possam ser devidas a variações genéticas (WEBER et al., 2000), HARTNOll et al. (1993) consideram que tais diferenças são também decorrentes do período necessário para atingir a muda puberal, quando a predação de espécimes pré-puberais com muda no maior tamanho reduz o tamanho máximo e médio dos espécimes pós-puberais. Além dessas interferências, LóPEz GRECO et al. (2000) consideram que o crescimento de $M$. bicornutus seja dependente da disponibilidade de espaço, da qualidade e da quantidade de alimento. Sem excluir a possibilidade de diferenciação genética, consideramos que os dados populacionais encontrados indicam que a predação e a competição intra e inter específica pelo alimento e pelo espaço do substrato, devem ser incluídos entre os moduladores do crescimento de $M$. bicornutus no fital $H$. opuntia.

Entre os Mithracidae tem sido indicado que, dependendo do tamanho e/ou do grau de maturação, a proporção entre machos e fêmeas pode ser variável. Enquanto resultados obtidos para Mithraculus forceps (A. Milne-Edwards, 1875) (MANTElatTo et al., 2003) e para $M$. bicornutus (LÓPEZ GRECO et al., 2000; CARMONA-SUÁREZ, 2007) mostraram que a razão sexual dessas espécies tende a ser de 1:1, para Hyas coarctatus Leach, 1815 e Inachus dorsettensis (Pennant, 1777) (HARTNOLl et al., 1993) é evidenciado um desvio favorável à predominância de fêmeas, durante a passagem da fase imatura para a madura. Os valores detectados em $H$. opuntia denotam que, independentemente do estágio de desenvolvimento sexual, a proporção entre os sexos é praticamente constante, exibindo significativa superioridade numérica de machos. WENNER (1972) considera que apesar da seleção natural favorecer a relação de 1:1 entre os sexos, esta proporção constitui uma exceção e não a regra entre os crustáceos marinhos maduros.

Embora não existam comprovações experimentais, o fato de $M$. bicornutus exibir no fital $H$. opuntia superioridade de machos, uma grande diferença entre o número de fêmeas imaturas e maduras e variações nos índices de razão sexual, indicam ocorrência de migração diferencial entre os sexos e/ou maior mortalidade de fêmeas. Apesar da agregação de fêmeas e machos maduros não ter sido evidenciada, o aumento do número de fêmeas ovígeras na estação de chuvas sugere que, durante este período, fêmeas maduras migrem para $H$. opuntia para realizarem o acasalamento e a desova.

O predomínio de juvenis em todos os pontos de coleta indica que, por apresentar fronde tridimensionalmente intrincada com formação de microhabitats, $H$. opuntia representa um habitat favorável ao desenvolvimento desta faixa etária, propiciando condições de moradia, abrigo e proteção dos espécimes, assim como constatado para outros braquiúros e invertebrados em diversos fitais (MonTouchet, 1979; Masunari, 1982; Dubiaski-Silva \& Masunari, 1995; Chemello \& Milazzo, 2002; Barbosa \& Leonel, 2003). Estando claramente relacionado com a entrada de juvenis em Picãozinho e em São Gonçalo, o aumento populacional estacional de $M$. bicornutus constitui uma indicação de que o ciclo reprodutivo desta espécie deve ser particularmente afetado pelo maior aporte de nutrientes durante o período chuvoso no Nordeste do Brasil.

Refletindo a estabilidade da temperatura da água, nenhuma interferência deste fator foi observada sobre a biomassa da alga ou sobre o número de espécimes, conforme observado por MUKAI (1971) em fital de clima temperado. Do mesmo modo, a ausência de correlação entre a salinidade da água e a biomassa da alga e o número de espécimes indica independência das variações populacionais observadas em relação a estes fatores. 
Apesar do pisoteio de algas coralíneas causar diminuição da biomassa por perda de tecido (BROwN \& TAYLOR, 1999), contrariamente ao esperado, nenhuma variação significativa deste parâmetro foi assinalada entre os pontos de coleta, incluindo as subáreas (Sul e Leste) de maior fluxo de pessoas. Uma vez que a biomassa de algas e gramas marinhas sofre uma diminuição proporcional à intensidade e à duração do pisoteio (BROwN \& TAYLOR, 1999; ECKRICH \& HOLMQUIST, 2000), a ausência de variação da biomassa de $H$. opuntia leva a supor que este resultado possa estar relacionado não apenas com a resistência da alga à fragmentação, mas também com a possibilidade dos bancos existentes em Picãozinho estarem submetidos a uma frequiência e intensidade de pisoteio variável. Como a metodologia adotada não permitiu uma observação continuada dos bancos de $H$. opuntia localizados no percurso realizado pelos visitantes, para maior esclarecimento dos aspectos mencionados, novos experimentos são recomendáveis.

Enquanto em São Gonçalo foram notadas maior constância e estabilidade dos valores de densidade populacional ao longo do tempo, as densidades determinadas em Picãozinho evidenciam que os aumentos populacionais observados nas subáreas Norte e Leste de Picãozinho estiveram nitidamente associados à entrada de juvenis no período chuvoso. Embora dados de correlação negativa entre o número de visitantes e de espécimes de M. bicornutus em Picãozinho Leste sejam indicativos de efeito antrópico negativo, este resultado deve ser visto com reservas uma vez que a diminuição do número de espécimes nos meses de estiagem estaria relacionada com o ciclo reprodutivo da espécie e não com o aumento do número de visitantes neste período.

Apesar das variações populacionais encontradas serem ainda pouco conclusivas quanto à influência da presença dos visitantes sobre o fital $H$. opuntia, os resultados de baixa densidade populacional e de maior desproporção da relação machos:fêmeas determinados na subárea Sul indicam que esta resposta pode ser resultante do pisoteio. Como em decorrência desta atividade, a diminuição da área superficial da plantasubstrato e da quantidade de sedimento retido na planta altera as condições de abrigo e de fornecimento de alimento, afetando a densidade populacional de diversas espécies (BROWN \& TAYLOR, 1999), admitimos que naquela subárea deve ocorrer uma migração mais intensa de fêmeas à procura de habitats mais propícios ao abrigo e alimentação da espécie. A migração para habitat denso localizado fora da rota de pisoteio é também considerada por ECKRICH \& HOLMQUIST (2000) como a explicação mais provável para a redução de diversas espécies de camarões associadas a T. testudinum. A hipótese de migração de fêmeas é ainda reforçada, ao considerarmos segundo KILAR \& LOu (1986), que estando incluídos entre os poucos invertebrados que participam da dieta alimentar sustentando o crescimento e a reprodução de $M$. bicornutus, os poliquetos estão entre os invertebrados mais sensíveis ao pisoteio de algas coralíneas (BROwn \& TAYLOR, 1999).

Os dados aqui obtidos indicam ocorrer variações populacionais similares quanto à composição dos espécimes em diferentes estágios de maturação, à freqüência de tamanho, à razão sexual e aos períodos reprodutivos de $M$. bicornutus em Picãozinho e São Gonçalo, sob influência dos mesmos parâmetros ambientais. No entanto, por diferirem dos resultados obtidos nos demais pontos de coleta, os dados de densidade populacional e de razão sexual determinados para a espécie ao Sul de Picãozinho são sugestivos de perturbações populacionais induzidas por pisoteio decorrente do fluxo de pessoas e/ou por fatores ambientais não analisados neste trabalho.

Agradecimentos. Ao Dr. Richard G. Hartnoll da Universidade de Liverpool pelos comentários e sugestões referentes aos critérios adotados na separação de machos imaturos e maduros, à Dra. Amélia Kanagawa pela identificação da alga Halimeda opuntia, à doutoranda Michelle Gomes Santos pelo apoio na análise estatística, ao geógrafo Utaiguara de Nóbrega Borges pela elaboração do mapa, ao Dr. Fernando Luis Medina Mantelatto e a Dra. Setuko Masunari pelas contribuições na revisão final deste trabalho. A CAPES, pela concessão da bolsa de estudo a Jefferson B. Batista e a Marcos Antonio J. Costa e ao WWFBrasil pelo apoio financeiro concedido.

\section{REFERÊNCIAS BIBLIOGRÁFICAS}

Alunno-Bruscia, M. \& Sainte-Marie, B. 1998. Abdomen allometry, ovary development, and growth of female snow crab, Chinoecetes opilio (Brachyura, Majidae) in the northwestern Gulf of St. Lawrence. Canadian Journal of Fisheries Aquatic Science 55:459-477.

Barbosa, J. H. A. J. \& Leonel, R. M. V. 2003. Sobre a comunidade de crustáceos em algas de entre-marés: Uma ênfase aos Brachyura. Cadernos Camilliani 4(1):19-29.

Brown, P. J. \& TAYLOR R. B. 1999. Effects of trampling by humans on animals inhabiting coralline algal turf in the rocky intertidal. Journal of Experimental Marine Biology and Ecology 235:45-53.

CARMONA-SuÁrez, C. 1992. Interpopulational size variation in a tropical decorator crab, Microphrys bicornutus (Latreille, 1825) (Decapoda, Brachyura, Majidae). Crustaceana 62:319-322.

2007. Spatial distribution, density, and relative growth of Microphrys bicornutus (Latreille, 1825) (Brachyura: Majidae) in five biotopes in a Thalassia complex. Scientia Marina 71(1):5-14

Chemello, R. \& Milazzo, M. 2002. Effect of algal architecture on associated fauna: some evidence from phytal mollusks. Marine Biology 140:981-990.

Coen, L. D. 1988. Herbivory by Caribbean majid crabs: feeding ecology and plant susceptibility. Journal of Experimental Marine Biology and Ecology 122:257-276.

Dubiaski-Silva, J. \& Masunari, S. 1995. Ecologia populacional dos Amphipoda (Crustacea) dos fitais de Caiobá, Matinhos, Paraná, Brasil. Revista Brasileira de Zoologia 12(2):373-396.

EcKrich, C. E \& Holmouist, J. G. 2000. Trampling in a seagrass assamblage: direct effects, response of associated fauna, and the role of substrates characteristics. Marine Ecology Progress Series 201:199-209.

EDGAR, G. J. 1983. The ecology of South-East Tasmanian phytal animal communities. I. Spatial organization on a local scale. Journal of Experimental Marine Biology and Ecology 70:129-157

GouvÊA, E. P. \& Leite, Y. M. 1980. A carcinofauna do fital de Halimeda opuntia (Linnaeus) Lamouroux e a variação sazona de sua densidade. Ciência e Cultura 32(5):596-600.

Grotta, M. \& Lunetta, J. E. 1980. Ciclo sexual de Anomalocardia brasiliana (Gmelin, 1791) do litoral do Estado da Paraíba. Revista Nordestina de Biologia 1(3):5-55.

Hartnoll, R. G. 1963. The biology of Manx spider crabs. Proceedings of the Zoological Society of London 141:423-496.

1965. The biology of spider crabs: A comparison of British and Jamaican species. Crustaceana 9:1-16.

Hartnoll, R. G.; Bryant, A. D. \& Gould, P. 1993. Size distribution in spider crab populations - Spatial and temporal variation. Journal of Crustacean Biology 13(4):647-655. 
KILAR, J. A. \& Lou, R. B. 1984. Ecological and behavioural studies of the decorator crab, Microphrys bicornutus Latreille (Decapoda: Brachyura): a test of optimum foraging theory. Journal of Experimental Marine Biology and Ecology 74:157-167.

1986. The subtleties of camouflage and dietary preference of the decorator crab, Microphrys bicornutus Latreille (Decapoda: Brachyura). Journal of Experimental Marine Biology and Ecology 101:143-160.

Jernakoff, P. \& Nielsen, J. 1998. Plant animal associations in two species of sea grasses in Western Australia. Aquatic Botany 60:359-376.

López Greco, L. S.; HernandéZ, J. E.; Bolaños, J.; Rodríguez, E. M. \& Hernandéz, G. 2000. Population features of Microphrys bicornutus Latreille, 1825 (Brachyura, Majidae) from Isla Margarita, Venezuela. Hydrobiologia 439:151-159.

Mantelatto, F. L. M.; Faria, F. C. R. \& Garcia, R. B. 2003. Biological aspects of Mithraculus forceps (Brachyura: Mithracidae) from Anchieta island, Ubatuba, Brazil. Journal of Marine Biological Association of the United Kingdom 83:789-791.

Masunari, S. 1982. Organismos do fital Amphiroa beauvoisi Lamouroux, 1816 (Rhodophyta: Corallinaceae), I. Autoecologia. Boletim de Zoologia da Universidade de São Paulo 7:57-148

1983. The phytal of the Alga Amphiroa fragilissima (Linnaeus) Lamouroux, 1816. Studies on Neotropical Fauna and Environment 18:151-161.

1987. Ecologia das comunidades fitais. In: SimpósIo SOBRE ecossistemas da costa Sul e Sudeste brasileira. Anais... Cananéia, Aciesp. v. 54(1). p.195-253.

Masunari, S. \& Forneris, L. 1981. O ecossistema fital - uma revisão. In: Seminários de Biologia Marinha. Anais... Rio de Janeiro. Aciesp. p.149-172.

Melo, G. A. S. 1996. Manual de identificação dos Brachyura (caranguejos e siris) do litoral brasileiro. São Paulo, Plêiade. 551p.

Melo, G. A. S. \& Veloso, V. G. 2005. The Brachyura (Crustacea, Decapoda) of the coast of the State of Paraíba Brazil, collected by Project Algas. Revista Brasileira de Zoologia 22(3):796805 .
Montouchet, P. C. G. 1979. Sur la communauté des animaux vagiles associes à Sargassum cymosum C. Agardh, à Ubatuba, Etat de São Paulo, Brésil. Studies on Neotropical Fauna and Environment 14:33-64

Moore, P. G. 1973. The nematode fauna associated with holdfasts kelp (Laminaria hyperborea) in North-East Britain. Journal of Marine Biological Association of the United Kingdom 51:589-604.

Mukai, H. 1971. The phytal animals on the thalli of Sargassum serratifolium in the Sargassum region, with reference to their seasonal fluctuations. Marine Biology 8:170-182.

NaKamura, Y.; Horinouchi, M.; NaKai, T. \& Sano, M. 2003. Food habits of fishes in a seagrass bed on a fringing coral reef at Iriomote Island, southern Japan. Ichthyological Research 50:15-22.

Nimer, E. 1977. Clima. In: Goldemberg, C. ed. Geografia do Brasil. Região Nordeste. Rio de Janeiro, Sergraf- IBGE. v.2. p.162-182

Palma, A. T. \& OJeda F. P. 2002. Abundance, distribution and feeding patterns of a temperate reef fish in subtidal environments of Chilean coast: the importance of understory algal turf. Revista Chilena de História Natural 75:189200

Parker, J. D.; Duffy, J. E. \& Orth, R. J. 2001. Plant species diversity and composition: experimental effects on marine epifaunal assemblages. Marine Ecology Progress Series 224:55-67.

Rocha, L. A.; Rosa, I. L. \& Rosa, R. S. 1998. Peixes recifais da costa da Paraíba, Brasil. Revista Brasileira de Zoologia 15(2):553-566.

Statsoft, inc. 1998. Statistica for Windows (Computer Program Manual). Tulsa. Oklahoma.

Van Alstyne, K. L.; Wolfe, G. V.; Freidenburg, T. L.; Neill, A. \& Hicken, C. 2001. Activated defense systems in marine macroalgae: evidence for an ecological role for DMSP cleavage. Marine Ecology Progress Series 213:53-65.

Weber, L. I.; Hartnoll, R. G. \& Thorpe, J. P. 2000. Genetic divergence and larval dispersal in two spider crabs (Crustacea: Decapoda). Hydrobiologia 420:211-219.

WenNer, A. M. 1972. Sex ratio as a function of size in marine crustacea. The American Naturalist 106(949):320-350. 